\title{
Cutaneous Complications Secondary to Haemostasis Abnormalities in COVID-19 Infection
}

\author{
Noel Lorenzo-Villalba ${ }^{1}$, Yasmine Maouche $^{1}$, Aneska Syrovatkova ${ }^{1}$, Felix Pham ${ }^{2}$, Jean-Baptiste Chahbazian ${ }^{1}$, \\ Pierre Pertoldi ${ }^{1}$, Emmanuel Andrès ${ }^{1}$, Abrar-Ahmad Zulfiqar ${ }^{1}$ \\ ${ }^{1}$ Service de Médecine Interne, Diabète et Maladies Métaboliques, Hôpitaux Universitaires de Strasboug, Strasbourg, France \\ ${ }^{2}$ Service de Dermatologie, Hôpitaux Universitaires de Strasboug, Strasbourg, France
}

Received: 02/06/2020

Accepted: 05/06/2020

Published: $12 / 06 / 2020$

How to cite this article: Lorenzo-Villalba N, Maouche Y, Syrovatknova A, Pham F, Chahbazian JB, Pertoldi P, Andrès E, Zulfiqar AA. Cutaneous complications secondary to haemostasis abnormalities in COVID-19 infection. EJCRIM 2020;7: doi:10.12890/2020_001769.

Conflicts of Interests: The Authors declare that there are no competing interests.

This article is licensed under a Commons Attribution Non-Commercial 4.0 License

\section{ABSTRACT}

We describe the case of a patient hospitalized for acute decompensated heart failure in a standard medical ward. During hospitalization, he was diagnosed with COVID-19 and transferred to a special unit. The clinical course was marked by worsening of the respiratory disease, the development of right parotiditis and thrombosis of the left internal jugular vein. Therapeutic anticoagulation was initiated and 2 days later, the minimal dermatoporosis lesions previously present in the upper extremities evolved to haemorrhagic bullae with intra-bullae blood clots and dissecting haematomas. Surgical management of the dissecting haematomas was difficult in the context of haemostasis abnormalities. The patient died 29 days after hospital admission.

\section{LEARNING POINTS}

- Single room accommodation should be preferred to double room accommodation in standard wards during the COVID-19 pandemic.

- Anticoagulation therapy and the presence of lupus anticoagulant may induce cutaneous complications during COVID-19 infection.

- The discontinuation of anticoagulation therapy did not help improve the management of cutaneous lesions.

\section{KEYWORDS}

COVID-19, dissecting haematoma, dermatoporosis, coagulation abnormalities

\section{INTRODUCTION}

There is currently little information about the prevalence and nature of dermatological manifestations related to COVID-19 infection. Isolated herpetiform lesions, petechial rash, erythematous rash, widespread urticaria and chickenpox-like vesicles have been reported. The trunk is the main region involved ${ }^{[1,2]}$.

\section{CASE DESCRIPTION}

An 84-year-old man was admitted to the emergency department for a 4-day history of general weakness and anorexia. No fever, cough or dyspnoea was reported. His medical history was relevant for hypertension, type 2 diabetes, chronic heart failure and chronic obstructive pulmonary disease. He was retired and lived alone.

Investigations in the emergency department showed elevated BNP of $1107 \mathrm{ng} / \mathrm{l}$ (NV <100 ng/l), a glomerular filtration rate of $74 \mathrm{ml} / \mathrm{min}$, normal electrolytes and white cell count, haemoglobin of $10.9 \mathrm{~g} / \mathrm{dl}$ and platelets at $58 \times 10^{9} / \mathrm{I}$ (NV 150-400 $\left.\times 109 / \mathrm{I}\right)$. The RT-PCR test on oropharyngeal swabs for SARS-CoV-2 was negative. CT scanning of the chest showed signs of vascular overload and absence of radiological signs consistent with COVID-19 infection. The patient was transferred to a double room in a standard medical ward and intravenous diuretics were initiated. 
Upon admission, the patient was afebrile, his blood pressure was 150/75 mmHg, heart rate was 78 bpm and oxygen saturation was $94 \%$ on room air. He was alert and oriented. Heart sounds were regular without any rubs or murmurs. Breath sounds were globally diminished with rales in both pulmonary fields. Minimal dermatoporosis lesions were noted on the four extremities.

On day 9, a new RT-PCR test on oropharyngeal swabs for SARS-CoV-2 was performed as the patient shared the room with a suspected COVID-19 patient. The PCR came back positive and he was transferred to the special unit.

On day 23, the patient presented a sudden painless bilateral cervical tumour. CT scanning of the neck confirmed the presence of right parotiditis associated with thrombosis of the left jugular vein (Fig. 1). The coagulation panel showed prothrombin time $76 \%$, TCA 51 sec (NV 25-42 sec), fibrinogen 6.55 g/l (NV 2-4 g/l), V factor normal, VIII factor 260\% (NV 60-150\%), anti-Xa antibodies and protein C normal, protein S 43\% (NV > 65\%), D-dimers $840 \mu \mathrm{g} / \mathrm{I}(\mathrm{NV}<500 \mu \mathrm{g} / \mathrm{I})$, and the presence of lupus anticoagulant at high titres. Anticoagulation therapy with low-molecular-weight heparin was promptly initiated.

On day 25, the minimal dermatoporosis lesions previously presented evolved in the upper extremities to haemorrhagic bullae. The management of haemorrhagic bullae was marked by the development of intra-bullae blood clots making care very difficult. These cutaneous lesions evolved to dissecting haematomas (Figs. 2 and 3) in the four extremities. Surgical treatment was initiated but discontinued owing to abundant bleeding. The clinical situation progressively worsened and the patient died 29 days after hospital admission.

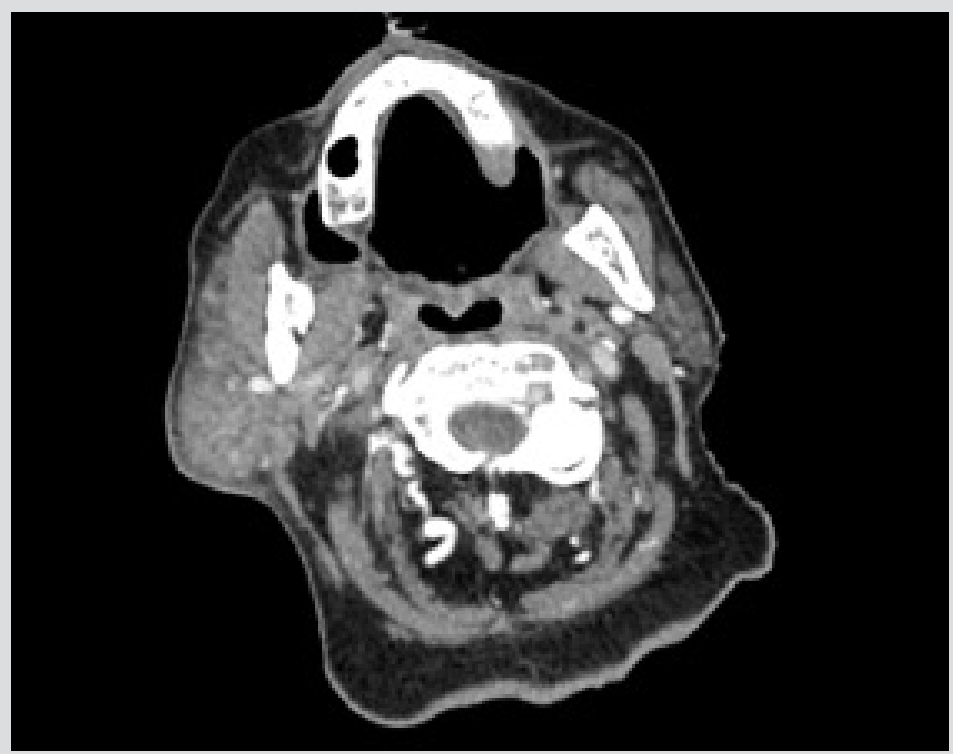

Figure 1. CT scan of the neck showing right parotiditis
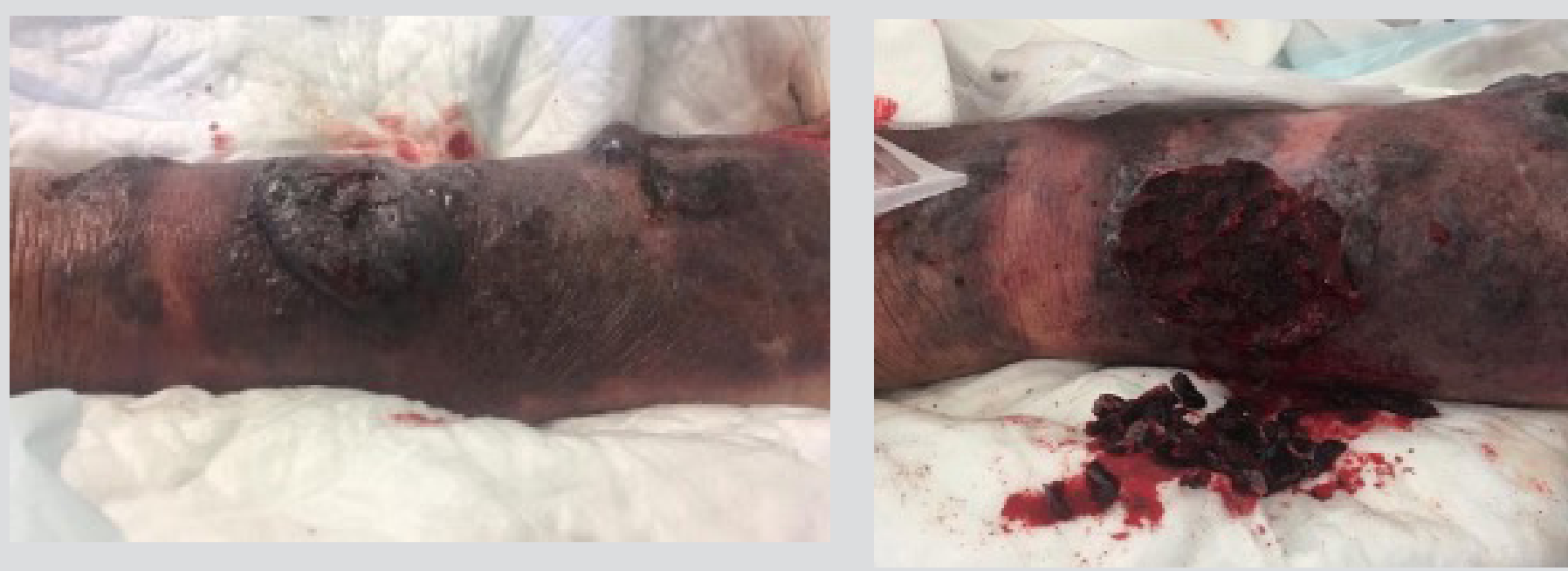

Figure 2 and 3. Dissecting ematoma 


\section{DISCUSSION}

Direct or indirect effects of COVID-19 infection, such as hypoxia, may predispose to thrombotic events. In addition, critical disease, the severe inflammatory state, and underlying common risk factors may all predispose to thrombotic events as described in previous virulent coronavirus outbreaks ${ }^{[3]}$. The most consistent haemostatic abnormalities reported during COVID-19 infection include thrombocytopenia and increased D-dimer levels. During the course of the disease and related to its severity, other abnormalities such as prolongation of prothrombin time (PT) and international normalized ratio (INR), and of thrombin time (TT), and variably a trend toward shortened activated partial thromboplastin time (aPTT) have been reported ${ }^{[4]}$. It is still not clear whether these haemostatic disturbances are specifically related to SARS-CoV-2 or result from the cytokine storm. Another important factor playing an important role in the pathophysiology of thrombosis related to COVID-19 is the presence of antiphospholipid antibodies, but this needs further investigation ${ }^{[5]}$.

A Chinese study reported that about $40 \%$ of hospitalized patients with COVID-19 were at high risk of thromboembolic events, but no data on the use of VTE prophylaxis or the incident VTE events were available ${ }^{[6]}$

The patient described developed deep venous thrombosis and required curative anticoagulation therapy. The pre-existing minimal dermatoporosis lesions progressed to dissecting haematomas due to multiple factors: the existing minimal dermatoporosis, anticoagulation therapy and coagulation abnormalities due to COVID-19 infection.

The incidence of dissecting haematoma is not known but is progressively increasing. Many risk factors such age (dermatoporosis), recent trauma, corticoid treatment, anticoagulation therapy (50\%: 30\% antiplatelet, $20 \%$ anti-vitamin $\mathrm{K}$ inhibitors) and coagulation disturbances have been related to this condition. Other comorbid conditions contributing to its development include diabetes, venous and arterial insufficiency, polyneuropathy, malnutrition and vitamin C deficiency.

Pathophysiologically, minimal trauma leads to bleeding in a virtual space between subcutaneous fat and muscle fascia. Pressurized blood dissects the virtual space between muscle and skin. Dissecting haematomas cause significant morbidity with the patient needing hospitalization and urgent surgical procedures as in the present case. Unfortunately, surgical treatment had to be discontinued due to abundant bleeding resulting from coagulation abnormalities.

\section{CONCLUSIONS}

Dissecting haematomas are rare. In the case presented, they resulted from a combination of various factors: minimal dermatoporosis, anticoagulation therapy and coagulation abnormalities due to COVID-19 infection.

\section{REFERENCES}

1. Tammaro A, Adebanjo GAR, Parisella FR, Pezzuto A, Rello J, Tammaro A, et al. Cutaneous manifestations in COVID-19: the experiences of Barcelona and Rome. J Eur Acad Dermatol Venereol 2020 Apr 24. doi: 10.1111/jdv.16530 [Epub ahead of print].

2. Recalcati S. Cutaneous manifestations in COVID-19: a first perspective. J Eur Acad Dermatol Venereol 2020;34(5):e212-e213.

3. Bikdeli B, Madhavan MV, Jimenez D, Chuich T, Dreyfus I, Driggin E, et al. COVID-19 and thrombotic or thromboembolic disease: implications for prevention, antithrombotic therapy, and follow-up. J Am Coll Cardiol 2020:S0735-1097(20)35008-7.

4. Lippi G, Salvagno GL, Ippolito L, Franchini M, Favaloro EJ. Shortened activated partial thromboplastin time: causes and management. Blood Coagul Fibrinolysis 2010;21:459463.

5. Zhang Y, Xiao M, Zhang S, Xia P, Cao W, Jiang W, et al. Coagulopathy and antiphospholipid antibodies in patients with Covid-19. N Engl J Med 2020;382(17):e38.

6. Wang T, Chen R, Liu C, Liang W, Gaun W, Tang R, et al. Attention should be paid to venous thromboembolism prophylaxis in the management of COVID-19. Lancet Haematol 2020;7(5):e362-e363.

7. Kaya G, Saurat JH. Dermatoporosis: a chronic cutaneous insufficiency/fragility syndrome. Clinicopathological features, mechanisms, prevention and potential treatments. Dermatology 2007;215(4):284-294. 